University of Zurich

Department of Economics

Working Paper Series

ISSN 1664-7041 (print)

ISSN 1664-705X (online)

Working Paper No. 112

\title{
Local contraction-stability and uniqueness
}

Andreas M. Hefti

February 2013 


\title{
Local contraction-stability and uniqueness*
}

\author{
Andreas M. Hefti ${ }^{\dagger}$
}

February 2013

\begin{abstract}
This paper investigates the relationship between uniqueness of Nash equilibria and local stability with respect to the best-response dynamics in the cases of sum-aggregative and symmetric games. If strategies are equilibrium complements, local stability and uniqueness are the same formal properties of the game. With equilibrium substitutes, local stability is stronger than uniqueness. If players adjust sequentially rather than simultaneously, this tends towards making a symmetric equilibria of symmetric games more stable. Finally, the relationship between the stability of the Nash best-response dynamics is compared to the stability of the response-dynamics induced by aggregate-taking behavior.
\end{abstract}

Keywords: Contraction mapping, Stability, Uniqueness, Aggregate-taking behavior, Dominance solvability, Symmetric Games

JEL Classification: C72, D43, L13

\footnotetext{
*I wish to thank Diethard Klatte, Armin Schmutzler and Josef Falkinger for their valuable comments.

${ }^{\dagger}$ Harvard University, Department of Economics, Littauer Center M-35, Cambridge, MA. E-mail: ahefti@fas.harvard.edu
} 


\section{Introduction}

Since Cournot's famous mathematical illustration of a stable equilibrium (Cournot (1897)), i.e. a situation where mutual rational, non-cooperative behavior dynamically restores a previous accidental departure of the equilibrium, the stability of an equilibrium has been a major concern to economists. It has been recognized early that the stability of an equilibrium depends critically on the underlying notion of dynamics (see e.g. McManus and Quandt (1961) for an early illustration), which are not generically specified by the conventional definition of a normal form game. Subsequent research revealed that examining dynamics matters not only on the conceptual ground of whether a (Nash)-equilibrium is eventually effectively reached when starting from a disequilibrium situation, but also because important mathematical connections between stability and other properties of the game have been uncovered. For example, Moulin (1984) finds a striking relationship between the stability of the equilibrium and whether a game is dominance-solvable or not in case of the stepwise best-reply adjustment process. In case of continuous adjustment process, where the individual rate of adjustment depends proportionally on the marginal benefit of playing the current strategy, Dixit (1986) highlights an intimate relationship between the stability of an equilibrium and the associated comparative static changes. Okuguchi and Yamazaki (2008) derive sufficient conditions for the unique equilibrium in sumaggregative games to be globally stable. Dastidar (2000) shows that there is a close relationship between local gradient stability and uniqueness of equilibria in the Cournot game. In this paper I pursue a similar question by investigating the relationship between uniqueness and local stability of the stepwise best-reply dynamics for the analytically tractable classes of sum-aggregative games and symmetric games. I show that if strategies are equilibrium complements (without the game e.g. being supermodular), local stability and uniqueness are the same mathematical properties for both classes of games, but local stability is only sufficient for uniqueness in case of substitutes. Further, it is shown that these results extend to local dominance-solvability and to aggregate-taking behavior, a type of behavior that has been subject to augmented research recently. In case of symmetric games it is illustrated that the difference between stability and uniqueness in dependence of the strategy type vanishes if we consider sequential rather than simultaneous updating of the best-replies, and economic implications of this finding are discussed. Moreover, it is shown that sum-aggregative symmetric games with substitutes naturally have a unique equilibrium, the symmetric equilibrium, which is necessarily stable under the gradient 
(or sequential) dynamics. This paper is organized as follows. After introducing the notation and general assumptions as well as the essential facts from matrix algebra, I derive the main results and tools required for the stability analysis in section 2. Although some results in this section are familiar, the derivation proceeds at a high level of generality and thereby keeps carefully track of the required assumptions. As newer results of matrix analysis are exploited, the reader may find proofs to be short, elegant and instructive. In section 3 I apply the previously developed machinery to sum-aggregative games for the case of conventional best-response and aggregate-taking behavior, and examine the relationship between uniqueness and stability. Section 4 provides a characterization when symmetric equilibria of (higher-dimensional) symmetric games are locally stable, and compares the relationship between uniqueness and stability for the simultaneous and sequential best-response dynamics and the gradient dynamics.

\section{Contractions and local contraction}

I first introduce the basic notation and essential mathematical concepts and then derive the main results about contractions, which are repeatedly used later in the paper.

\subsection{The game: Notation and assumptions}

Consider a game of $N \geq 2$ players. Specifically, the joint strategy space is $\mathcal{S} \equiv \mathcal{S}(k)=\prod_{j=1}^{N} S^{j}(k)$, where $S^{g}(k) \subset \mathbb{R}^{k}$, the strategy space space of player $g$, is non-empty and convex. $\operatorname{Int}(\mathcal{S})$ denotes the interior of $\mathcal{S}$. The point $x_{g} \equiv\left(x_{g 1}, \ldots, x_{g k}\right) \in S^{g}(k)$ denotes a strategy of player $g$ and $x=\left(x_{1}, \ldots, x_{N}\right) \in \mathcal{S} \subset \mathbb{R}^{k N}$ is a strategy profile. From the perspective of a player $g$ let $\mathcal{S}_{-g}$ denote the joint strategy space of all opponents of $g$ and $x_{-g} \in \mathcal{S}_{-g}$ is a particular strategy profile of $g$ 's opponents. The payoff of player $g$ is represented by $\Pi^{g} \in C^{2}(\mathcal{S}, \mathbb{R})$, where I always assume $\Pi^{g}$ to be strongly quasiconcave ${ }^{1}$ in $x_{g}$. Hence $\left(N, \mathcal{S}(k),\left\{\Pi^{j}\right\}\right)$ is a twice differentiable $k$-dimensional $N$-player game, simply referred to as a game. For such a game the joint best-response $\phi(x)=\left(\varphi^{1}\left(x_{-1}\right), \ldots, \varphi^{N}\left(x_{-N}\right)\right)$ is a well-defined and continuous function, i.e. $\phi \in C^{0}(\mathcal{S}, \mathcal{S})$. For notational simplicity I frequently write $\varphi^{j}(x)$ instead of $\varphi^{j}\left(x_{-j}\right)$. Let $\partial \phi(x)$ denotes its derivative at $x$. Finally, $\nabla \Pi(x) \equiv\left(\nabla_{j} \Pi^{j}(x)\right)_{j=1}^{N}$ is the $N k$-vector obtained by stacking all player gradients, and its Jacobian is denoted by $H(x)=\frac{\partial \nabla \Pi(x)}{\partial x}$.

\footnotetext{
${ }^{1}$ Strong quasiconcavity means that the Hessian $\frac{\partial^{2} \Pi^{g}(x)}{\partial x_{g} \partial x_{g}^{T}}$ is negative definite on $\left\{z \neq 0: z \cdot \frac{\partial \Pi(x)}{\partial x_{g}}=0, z \cdot z=1\right\}$ (see Diewert et al. (1981), p. 409).
} 


\subsection{Contraction mappings}

$X=\left(\mathbb{R}^{n},|\cdot|_{X}\right)$ and $Y=\left(\mathbb{R}^{m},|\cdot|_{Y}\right)$ are two complete metric spaces, where $|\cdot|$ denotes the respective vector norm. $\bar{U}$ denotes a compact and convex subset of $\mathbb{R}^{n}$ with non-empty interior $U$. Any reference e.g. to open or closed subsets of $\bar{U}$ means open or closed relative to $\bar{U}$ in the usual topological sense. Specifically, $\left(\bar{U},|\cdot|_{X \mid \bar{U}}\right)$ is another complete metric space.

Among the class of continuous functions, the subclass of Lipschitz-continuous function plays an outstanding role in economics. A function $\phi: \bar{U} \rightarrow \mathbb{R}^{m}, \bar{U} \subset \mathbb{R}^{n}$, is Lipschitz on $\bar{U}$ if there is $q>0$ such that $\left|\phi(x)-\phi\left(x^{\prime}\right)\right|_{Y} \leq q\left|x-x^{\prime}\right|_{X}$ for any $x, x^{\prime} \in \bar{U}$. Lipschitz-continuity arises naturally in many economic applications, as continuously differentiable functions on convex sets are locally Lipschitz, and Lipschitz if these sets are also compact.

Fact 1 If $\left(N, \mathcal{S},\left\{\Pi^{j}\right\}\right)$ is a game, $\phi(\mathcal{S}) \subset \operatorname{Int}(\mathcal{S})$ and $\mathcal{S}$ is compact, then $\phi$ is Lipschitz.

Proof:

Because $\phi(\mathcal{S}) \subset \operatorname{Int}(\mathcal{S})$, the Implicit Function Theorem $(\mathrm{IFT})$ asserts $\phi \in C^{1}(\mathcal{S}, \mathcal{S})$, which implies that $\phi$ is locally Lipschitz. Consequently, $\phi$ is Lipschitz if $\mathcal{S}$ is compact.

The function $\phi$ is called a contraction (mapping) if it is Lipschitz with $q<1$. The set of all contractions from $X$ to $Y$ are denoted as $\mathcal{C}(X, Y)$. It is important to bear in mind that contractions are always defined contingent on certain norms (as $X, Y$ are spaces rather than sets), and the contraction property generally is not invariant under equivalent norms ${ }^{2}$.

Contractions are the main ingredient of the Banach fixpoint (FP) theorem. Its beauty stems from the fact that it asserts the three desirable properties of a game, existence and uniqueness of equilibrium as well as global stability of the best-reply map, to simultaneously occur: If $\phi: \mathcal{S} \rightarrow \mathcal{S}$ is a contraction and $\mathcal{S}$ is compact, then $\phi$ has exactly one FP $x^{*}$ and the recurrence relation $x^{t}=\phi\left(x^{t-1}\right)$ converges globally (i.e. for any initial value $x^{0} \in \mathcal{S}$ ) to $x^{*}$. In memoriam of its initial discoverer, such convergence of the joint best-reply has frequently been quoted as Cournot stability.

\footnotetext{
${ }^{2}$ This is a crucial difference to the more general Lipschitz property, which is preserved under equivalent norms.
} 


\subsection{Essential facts from matrix analysis}

$M_{n}$ denotes the set of all real $n \times n$ matrices and $\lambda$ is an eigenvalue of $A \in M_{n} . \sigma(A)$ is the spectrum of $A$, i.e. the $n$-list of all eigenvalues of $A$ with corresponding spectral radius $\rho(A) \equiv \max \{|\lambda|: \lambda \in \sigma(A)\}$. Matrix norms are denoted by $\|\cdot\|$. A matrix norm has all properties of a vector norm and additionally satisfies submultiplicativity (i.e. $\|A B\| \leq\|A\|\|B\|$ ). Whereas some authors do not include submultiplicativity in their definition of a matrix norm, it is of central importance to this paper as under submultiplicativity the spectral radius gives a lower bound for any matrix norm. Mathematically, this holds as submultiplicativity imposes a restriction on which linear combinations of matrix norms generate new matrix norms. ${ }^{3}$ An important class of matrix norms are those induced by some vector norm $|\cdot|:\|A\|_{|\cdot|} \equiv \max _{|v|=1}|A v|$. As $\|A\|_{|\cdot|}$ turns the space of all bounded linear operators from $X$ to $Y$ into a Banach space (as $Y$ is a Banach space), such norms are frequently referred to as operator norms. The following facts about matrix norms and their spectra are well known (see e.g. Horn and Johnson (1985)):

Fact 2 For any matrix norm there exists an operator norm $\|\cdot\|_{|\cdot|}$ such that $\|A\|_{|\cdot|} \leq\|A\|$ is true for any $A \in M_{n}$. Moreover, we have that $|A v| \leq\|A\|_{|\cdot|}|v|$.

Fact 3 If $A \in M_{n}$ then $\rho(A) \leq\|A\|$ for any matrix norm. Moreover, for any $A \in M_{n}$ and any $\varepsilon>0$ there exists a matrix norm such that $\|A\|<\rho(A)+\varepsilon$. Furthermore, $\lim _{t \rightarrow \infty} A^{t}=0$ if and only if $\rho(A)<1$.

\subsection{Characterization of (local) contractions}

The following theorem provides a general characterization for a differentiable mapping $\phi$ to be a contraction. Notably, $\phi$ is not required to be continuously differentiable, nor differentiable at boundary points, and the linear operator $\partial \phi$ is not required to be a square matrix.

Theorem 1 Suppose $\phi \in C^{0}\left(\bar{U}, \mathbb{R}^{m}\right)$ is (Frechet)-differentiable on $U$. Then $\phi \in \mathcal{C}(\bar{U}, Y)$ if and only if

$$
\sup _{x \in U}\|\partial \phi(x)\|_{|\cdot|_{X}}<1
$$

Proof: Section 6.1

The fact that $\phi$ is continuous on $\bar{U}$ implies that (1) must be verified only for interior points of

\footnotetext{
${ }^{3}$ If $\|\cdot\|$ is a matrix norm, then $r\|\cdot\|$ is a matrix norm (i.e. satisfies submultiplicativity) if and only if $r \in[1, \infty)$.
} 
$\bar{U}$, which is convenient for applications as $\phi$ may not be differentiable on boundary points (see e.g. the rent-seeking game in section 3 ). Theorem 1 says that $\phi$ is a contraction if and only if its directional derivatives, i.e. its local rate of change in some direction $v$, are smaller than one as measured by the $|\cdot|_{Y}$-norm. In terms of minimal assumptions the proof of theorem 1 shows that compactness of $\bar{U}$ is not critical, but differentiability and convexity are vital assumptions. In economic theory, the case $Y=X$ is of central importance, mainly because then the Banach FP theorem can eventually be applied. If the premise of theorem 1 is satisfied then fact 2 in conjunction with theorm 1 implies that if a matrix norm $\|\cdot\|$ satisfies $\|\partial \phi(x)\|<1$ for any $x \in U$, then there is a norm $|\cdot|_{X}$ such that $\phi$ is a contraction on $\bar{U}$.

Under the additionally assumption that $\phi$ is continuously differentiable on $\bar{U}$, we obtain a condition on the spectral radius of $\partial \phi(x)$ :

Corollary 1 Suppose that $Y=X$ and $\phi \in C^{1}\left(\bar{U}, \mathbb{R}^{n}\right)$. Then there exists a norm $|\cdot|$ such that $\phi \in \mathcal{C}\left(\bar{U}, \mathbb{R}^{n}\right)$ if and only if $\sup _{x \in U} \rho(\partial \phi(x))<1$.

Proof: Section 6.2

I now derive a local version of when a function is a contraction. Suppose that $\phi: \bar{U} \rightarrow \bar{U}$ and let $x^{*}$ be a $\mathrm{FP}$ of $\phi$. I say that $x^{*}$ is (locally) contraction-stable if there is a compact, convex and complete metric space $(V,|\cdot|), x^{*} \in V \subset \bar{U}$, such that $\phi \in \mathcal{C}(V, V)$.

Theorem 2 (Local contractions) Suppose $\phi: \bar{U} \rightarrow \bar{U}, \phi\left(x^{*}\right)=x^{*}$ and $\partial \phi$ is continuous at $x^{*}$. Then $x^{*}$ is contraction-stable if and only if

$$
\left\|\partial \phi\left(x^{*}\right)\right\|_{|\cdot|}<1
$$

Moreover, if (2) is satisfied, the best-reply process $x^{t}=\phi\left(x^{t-1}\right)$ converges to $x^{*}$ for any initial value $x^{0} \in V$.

Proof: Section 6.3

The next two results follow from theorem 2 and fact 3 .

Corollary 2 Under the premise of theorem 2 we can replace (2) by the requirement that $\rho\left(\partial \phi\left(x^{*}\right)\right)<$ 1. 
Examples can be constructed, where the best-reply dynamics $\left(x_{t}\right)$ converges to $x^{*}$ even if $\rho\left(\partial \phi\left(x^{*}\right)\right)=1$. This is a knife-edge case as there is no neighborhood ${ }^{4} V$ of $x^{*}$ such that $x^{t} \rightarrow x^{*}$ on $V$ if $\phi$ is a local expansion $\left(\rho\left(\partial \phi\left(x^{*}\right)\right)>1\right)$. Clearly, the best-response $\phi$ clearly cannot be a local contraction if $\rho\left(\partial \phi\left(x^{*}\right)\right)=1$. Moreover, theorem 4 in section 3.1 shows that if $\rho\left(\partial \phi\left(x^{*}\right)\right)=1$ at an equilibrium candidate, then $\operatorname{Det}\left(H\left(x^{*}\right)\right)=0$, i.e. the game cannot be regular. But we cannot hope to disentangle the connection between uniqueness and stability in general for non-regular games. In fact, the notion of contraction-stability is precisely what is required to describe the sharp mathematical relationship between uniqueness and stability. Finally, if there is local convergence and $\rho\left(\partial \phi\left(x^{*}\right)\right)=1$, the best-response $\phi$ cannot be linear at $x^{*}:$

Corollary 3 If $\partial \phi$ is continuous at the FP $x^{*}$, then $x^{*}$ is contraction-stable if and only if $\phi$ 's linearization at $x^{*}, L(x)=\partial \phi\left(x^{*}\right) x+\left(I-\partial \phi\left(x^{*}\right)\right) x^{*}$, is a contraction on $\mathbb{R}^{n}$. Moreover, the speed of convergence is approximately bounded by $\frac{\left|x_{t+1}-x^{*}\right|}{\left|x_{0}-x^{*}\right|} \leq \rho\left(\partial \phi\left(x^{*}\right)\right)^{t}$.

\section{Proof:Section 6.4}

Note from the convergence rate inequality that the spectral radius not only qualitatively determines whether or not a FP is contraction-stable but also influences how quickly a certain vicinity of the FP is reached; a smaller value suggests that fewer iterations are required.

It is also noteworthy that contraction-stability is invariant to forward-anticipation of the players, as long as all players anticipate in the same way. ${ }^{5}$ If all players forward-anticipate the bestresponse of their opponents e.g. by $r=1$ period, the best-reply process corresponds to $x^{t+1}=$ $\phi \circ \phi \circ\left(x^{t}\right)=\phi^{3}\left(x^{t}\right)$, or generally to $x^{t+1}=\phi^{2 r+1}\left(x^{t}\right)$ for $r$ steps.

Proposition 1 The composition $\phi^{(2 r+1)}$ is a local contraction at $x^{*}$ if and only if $\phi$ is a local contraction at $x^{*}$.

\section{Proof:}

Evaluating the adjustment matrix of $\phi^{2 r+1}(x)$ at $x^{*}$ gives $\partial \phi\left(x^{*}\right)^{2 r+1}$, which by fact 3 and corollary 2 implies the result.

\footnotetext{
${ }^{4}$ If $\phi$ is a diffeomorphism on $\mathbb{R}^{n}$ and $x^{*}$ is a hyperbolic FP $\left(|\lambda| \neq 1 \forall \mathrm{EV}\right.$ of $\left.\partial \phi\left(x^{*}\right)\right)$ then even if $\rho\left(\partial \phi\left(x^{*}\right)\right)>1$ as long as at least one EV has $|\lambda<1|$, there can be some lower-dimensional submanifold about $x^{*}$ on which the best-reply dynamics converge to $x^{*}$ (see e.g. Nitecki (1971)).

${ }^{5}$ Section 4.2 examines the case of sequential adjustments.
} 
Despite its innocent looking nature, condition (2) (or its spectral counterpart) can be hard to verify in applications. Using particular induced matrix norms results in sufficient conditions on the row (for $|\cdot|_{\infty}$ ) or column (for $|\cdot|_{1}$ ) sums. Let $R_{m}\left(x^{*}\right) \equiv \sum_{t=1}^{N k}\left|\frac{\partial \varphi_{m}}{\partial x_{t}}\right|<1$ denote the $m$-th row sum of $\partial \phi\left(x^{*}\right)$ and $C_{m}\left(x^{*}\right) \equiv \sum_{t=1}^{N k}\left|\frac{\partial \varphi_{t}}{\partial x_{m}}\right|<1$ denotes the $m$-th column sum.

Corollary 4 If $\partial \phi$ is continuous at the FP $x^{*}$ and $R_{m}\left(x^{*}\right)<1$ or $C_{m}\left(x^{*}\right)<1$ holds $\forall m=$ $1, \ldots, N k$ then $x^{*}$ is a locally contraction stable equilibrium.

If $\phi \in C^{0}\left(\bar{U}, \mathbb{R}^{n}\right)$ and $R_{m}(x)<1$ (or $C_{m}(x)<1$ ) hold for any $x \in U$, then the Banach FP theorem together with theorem 1 assert that the game has a unique and globally contractionstable equilibrium.

For example, Hadar (1966) uses $\|\cdot\|_{|\cdot|_{\infty}}$ to discuss the scope of contraction-stability in an oligopolistic model of product differentiation, noting that diagonal-dominance is far from necessary. In case of sum-aggregative games it is possible to tractably generalize the stability conditions up to almost necessary conditions.

A straightforward application of the IFT shows that, for $k=1$, the Hadar-condition $\|\partial \phi(x)\|_{|\cdot|_{\infty}}<$ 1 is equivalent to the requirement that $H(x)$ has a dominant negative diagonal. This equivalence breaks down if $k>1$. It is easy to give numerical examples e.g. if $k=2$, where $R_{m}\left(x^{*}\right)<1$ ist true for all $m$ but $H\left(x^{*}\right)$ violated diagonal dominance. Nevertheless, the following result holds:

Corollary 5 Suppose $k=2, x^{*}$ is a FP and $H\left(x^{*}\right)$ has a dominant negative diagonal. Then $R_{m}\left(x^{*}\right)<1, m=1, \ldots, 2 N$.

Proof: See section 6.5

\section{Sum-Aggregative games}

Consider a one-dimensional game with $\Pi^{j}=\Pi^{j}\left(x_{j}, \sum x_{i}\right)$. The most intensively studied example of such a game is the Cournot model of quantity competition: $\Pi^{j}=P\left(\sum x_{i}\right) x_{j}-c^{j}\left(x_{j}\right)$, but also the CES-model of price-competition $\Pi^{j}=\left(x_{j}-c_{j}\right) \frac{x_{j}^{-\sigma}}{\sum_{i} x_{i}^{1-\sigma}} V_{j}$, where $x_{j}$ denotes player $j$ 's price, has been used in various applications of oligopolistic competition. More recently, rentseeking games of the form $\Pi^{j}=\pi\left(\frac{x_{j}}{\sum x_{i}+r_{j}}\right)-c^{j}\left(x_{j}\right)$ have received considerable attention (see Konrad (2009)). In the following two sections I derive the relationship between uniqueness 
and (local) contraction-stability of equilibria in such games for conventional Nash behavior and aggregate-taking behavior.

\subsection{Uniqueness and local contraction-stability}

I begin by deducing a useful characterization of contraction-stability in sum-aggregative games. Suppose that $\phi(\mathcal{S}) \subset \operatorname{Int}(\mathcal{S})$. Then for any $x \in \operatorname{Int}(\mathcal{S})$ the matrix $\partial \phi(x)$ takes on the form

$$
A=\left(\begin{array}{ccccc}
a_{1} & b_{1} & \cdots & \cdots & b_{1} \\
b_{2} & a_{2} & b_{2} & \cdots & b_{2} \\
\cdots & \cdots & \cdots & \cdots & \cdots \\
b_{N} & \cdots & \cdots & \cdots & a_{N}
\end{array}\right)
$$

where $a_{j}=0$ and $b_{j}=-\frac{\Pi_{12}^{j}+\Pi_{22}^{j}}{\Pi_{11}^{j}+2 \Pi_{12}^{j}+\Pi_{22}^{j}}$.

Lemma 1 If $a_{j} \neq b_{j}$ the matrix (3) has $\operatorname{Det}(A)=\prod_{j=1}^{N}\left(a_{j}-b_{j}\right)\left(\sum_{j=1}^{N} \frac{b_{j}}{a_{j}-b_{j}}+1\right)$. If $a_{j}=b_{j}$ for at least one $j$, then $\operatorname{Det}(A)=a_{j} \prod_{i \neq j}\left(a_{j}-b_{j}\right)$.

Proof:

If $a_{j} \neq b_{j}$ subtract the first column from all other columns, multiply out $\left(a_{j}-b_{j}\right)$ row-wise, add to the first row all other rows and calculate the determinant of the remaining lower-triangular matrix. If $a_{j}=b_{j}$ for more than one $j, \operatorname{Det}(A)=0$. If $a_{j}=b_{j}$ for exactly one $j$ we can assume without loss of generality that $j=1$, and subtracting the first column from all other columns gives a lower-triangular matrix.

From lemma 1 we get that an $\operatorname{EV} \lambda \neq 0$ of $\partial \phi(x)$ either solves $\sum \frac{b_{j}}{\lambda+b_{j}}=1$ or $^{6} \lambda=-b_{j}$.

Theorem 3 Let $x^{*}$ be an interior equilibrium of the sum-aggregative game as described above. If all $b_{j}\left(x^{*}\right)$ have the same sign and $\sum \frac{\left|b_{j}\left(x^{*}\right)\right|}{1+\left|b_{j}\left(x^{*}\right)\right|}<1$, then $x^{*}$ is contraction-stable. Moreover, if this holds at any $x \in \operatorname{Int}(\mathcal{S})$, then the game has a unique and globally contraction-stable equilibrium $x^{*}$.

\footnotetext{
${ }^{6}$ The second case can occur only if $b_{j}=b_{g}$ for at least two players.
} 
Proof:

Note that if $b_{j}\left(x^{*}\right) \geq 0$ for all $j, \rho\left(\partial \phi\left(x^{*}\right)\right) \geq 0$ is in fact an eigenvalue of $\partial \phi\left(x^{*}\right)$ according to the Perron-Frobenius theorem, and obviously $\rho\left(\partial \phi\left(x^{*}\right)\right) \neq-b_{j}\left(x^{*}\right)$. If $\sum \frac{b_{j}}{1+b_{j}}<1$ then $\sum \frac{b_{j}}{\lambda+b_{j}}=1$ implies that $\rho\left(\partial \phi\left(x^{*}\right)\right)<1$. If $b_{j}\left(x^{*}\right)<0$ the claim follows from the previous result as $-\partial \phi\left(x^{*}\right)$ is positive. The last claim follows from corollary 1 and the Banach FP theorem.

Remarkably, the conditions of theorem 3 are more general in case of strategic complements or substitutes (either $b_{j} \geq 0$ or $b_{j}<0 \forall j$ ) than the $R_{m^{-}}$or $C_{m^{-}}$-condition in corollary 4 .

Proposition 2 If $R_{m}\left(x^{*}\right)<1$ or $C_{m}\left(x^{*}\right)<1 \forall m$ and strategies are either complements or substitutes, then the premise of theorem 3 is satisfied and $x^{*}$ is contraction-stable.

Proof:

If $b_{j} \geq 0 \forall j$ and $R_{m}<1 \forall m$, then $b_{j}<\frac{1}{N-1}$ and hence $\sum_{j} \frac{b_{j}}{1+b_{j}}<1$. If $b_{j} \geq 0 \forall j$ and $C_{m}<1 \forall m$ then assuming without loss of generality that $b_{n} \geq b_{n-1} \geq \ldots \geq b_{1}$ implies $\sum_{j} \frac{b_{j}}{1+b_{j}} \leq \frac{1}{1+b_{1}} \sum_{j} b_{j}$ and the claim follows as $\sum_{j \neq 1} b_{j}<1$. The case of substitutes is proved similarly.

It is easy to find numerical examples showing the converse to be false. Moreover, if all $b_{j}$ have the same sign, $\sum \frac{\left|b_{j}\right|}{1+\left|b_{j}\right|}<1$ is necessary for contraction-stability, i.e. theorem 3 characterizes contraction-stability for such games:

Corollary 6 Suppose $x^{*}$ is an interior contraction-stable equilibrium of a sum-aggregative game and all $b_{j}\left(x^{*}\right)$ have the same sign. Then $\sum \frac{\left|b_{j}\left(x^{*}\right)\right|}{1+\left|b_{j}\left(x^{*}\right)\right|}<1$.

Proof:

If $b_{j}\left(x^{*}\right) \geq 0$ for all $j, \rho\left(\partial \phi\left(x^{*}\right)\right)$ is the largest EV of $\partial \phi\left(x^{*}\right)$ and $\sum_{j} \frac{b_{j}}{\rho+b_{j}}=1$ together with $\rho<1$ imply that $\sum_{j} \frac{b_{j}}{1+b_{j}}<1$. If $b_{j}\left(x^{*}\right)<0$ then the claim follows from a similar argument.

We also see from theorem 3 and corollary 6 that contraction-stability does not necessarily require individual slopes to satisfy $\left|b_{j}\right|<1$, nor is $\left|b_{j}\right|<1 \forall j$ itself sufficient for contraction-stability. But it is clear that at most one player $j$ can have $\left|b_{j}\left(x^{*}\right)\right| \geq 1$ for $x^{*}$ to be contraction-stable. 
If the slope condition in theorem 3 holds globally, then uniqueness and (global) stability of the equilibrium both hold simultaneously. Obviously, this condition is restrictive and often is violated in examples. But what can be said about the relationship between uniqueness and stability, if we require only local contraction-stability?

Concerning uniqueness of equilibria the index theorem (see e.g. Vives (1999)) tells us that a game with only regular interior equilibria has a unique equilibrium if and only if $\operatorname{Det}(-H(x))>0$ on $C r \equiv\{x \in \operatorname{Int}(\mathcal{S}): \nabla \Pi(x)=0\}$, where $C r$ is the set of critical points. I refer to a game with $\phi(\mathcal{S}) \subset \operatorname{Int}(\mathcal{S})$ and $\operatorname{Det}(H(x)) \neq 0$ on $C r$ as an index game. The main analytical power of the index theorem stems from the fact that it is very general and provides us with a local condition for uniqueness. The index characterization result together with the (local) characterization of contraction-stability (theorem 3 and corollary 6) allows us to give a remarkably simple proof that the following relationship between uniqueness and contraction-stability is satisfied:

Theorem 4 Consider a sum-aggregative index game. If $b_{j}(x) \geq 0 \forall j$ on $C r$, then the game has a unique equilibrium if and only if every $x \in C r$ is contraction-stable. If $b_{j}(x) \leq 0 \forall j$ on $C r$ and every $x \in C r$ is contraction-stable, then there is a unique equilibrium.

\section{Proof: Section 6.7}

Note that the premise of theorem 4 restricts the best-response slopes only on $\mathrm{Cr}$. Individual replies can be non-monotonic and have different signs outside of this set, as is the case in the rent-seeking example. In particular, the game needs not be super- nor submodular. Theorem 4 shows that uniqueness and contraction-stability are the same formal properties of a game with equilibrium complements. This is interesting as the index refers to a topological-algebraic property of a vector field, whereas contraction is a metric-analytical property of a function. Nevertheless, the restrictions imposed by the respective conditions of these theorems are the same in case of a (weakly) increasing joint-best response at critical points. A further consequence of theorem 4 is, that whenever a game with either equilibrium complements or substitutes has multiple equilibria, there must be unstable equilibria.

In case of equilibrium substitutes uniqueness and contraction-stability generally are not the same properties, uniqueness generally being less restrictive than contraction-stability. This is a difference to the gradient dynamics (see section 4.2) as used e.g. in Dastidar (2000), where it is shown that a unique equilibrium in the Cournot-model is almost always stable. The difference 
between substitutes and complements stems from the fact that both uniqueness and contractionstability in games with complements impose the same restriction on the collective equilibrium response, but uniqueness mainly imposes a restriction on individual slopes in case of substitutes. Moulin (1984) shows that there is a tight relationship between contraction-stability and local dominance-solvability of equilibria. A game is locally dominance solvable at $x^{*}$ if there exists a neighborhood $V\left(x^{*}\right)$ such that the individual successive elimination of dominated strategies shrinks the joint strategy to the singleton $\left\{x^{*}\right\}$ on $V\left(x^{*}\right)$. Let $\partial \phi\left(x^{*}\right)^{+}$denote the matrix derived from $\partial \phi\left(x^{*}\right)$ by replacing each entry with its absolute value. For a one-dimensional game we have that $x^{*}$ is locally dominance-solvable if and only if $f^{7} \rho\left(\partial \phi\left(x^{*}\right)^{+}\right)<1$. As $\rho(\partial \phi(x)) \leq \rho\left(\partial \phi(x)^{+}\right)$contraction-stability is less restrictive than dominance-solvability but in case of equilibrium complements or substitutes (i.e. all non-zero entries of $\partial \phi\left(x^{*}\right)$ have the same sign) the two properties are the same. Thus in view of theorem 4 we may immediately conclude that uniqueness in sum-aggregative index games with equilibrium complements always implies local dominance-solvability (and vice-versa). Similarly, if all critical points are contraction-stable in a sum-aggregative game with substitutes, then there is a unique and locally dominance-solvable equilibrium (and vice-versa).

As a final remark it should be noticed that we can use the above analysis to address the relationship between uniqueness and contraction-stability in two-player (not necessarily sumaggregative) games:

Proposition 3 A two-player index game where strategies are either complements or substitutes on $\mathrm{Cr}$ has a unique equilibrium if and only if every critical point is contraction-stable (or equivalently locally dominance-solvable).

\section{Proof:}

In view of theorem 4 we only have to prove that uniqueness implies contraction-stability in case of substitutes at $x \in C r$. If $b_{j}(x) \in(-1,0]$, then $\frac{b_{j}(x)}{b_{j}(x)-1} \in\left[0, \frac{1}{2}\right)$, hence $x$ is contraction-stable. If $b_{1}(x)=-1$ and $b_{2}(x) \in(-1,0]$, then $x$ also is contraction-stable. Finally, if $b_{1}(x)<-1$ and $b_{2}(x) \in(-1,0]$ we have $\operatorname{Det}(-H(x))>0$ if and only if $b_{1}(x)>\frac{1}{b_{2}(x)}$, which in turn is equivalent to $\sum \frac{b_{j}(x)}{b_{j}(x)-1}<1$, showing that $x$ is contraction-stable.

\footnotetext{
${ }^{7}$ Similar to the discussion in section 2.4, knife-edge cases can be constructed, where $x^{*}$ is locally dominancesolvable but $\rho\left(\partial \phi\left(x^{*}\right)^{+}\right)=1$. But then the type of game considered by theorem 3 would have a singularity at $x^{*}$, meaning that the index theorem cannot be invoked. Therefore, this non-generic case is ruled out by assumption.
} 


\subsection{Aggregate-taking behavior dynamics and uniqueness}

Recently, there has been increased interest in aggregate-taking behavior (ATB, see e.g. AlosFerrer and Ania (2005)) as opposed to Nash-behavior (NB). A relative advantage of ATB over NB is that analyzing a game under ATB usually is simpler in applications, especially in presence of heterogeneity (see e.g. Grossmann et al. (2012)). ATB means that the players play a bestresponse but not directly to the individual strategies of their opponents but rather to some aggregate strategy. For example, the players might ignore the exact composition of the aggregate because of perceptional or informational limitations. Although the concept of ATB is not limited to sums, the case of sum-aggregative games is natural in many settings and simple, explaining why it arises frequently in applications. The crucial point where ATB and NB differ is that under ATB the players ignore how their own strategy affects the aggregate when making their decisions. This points towards a more Walrasian motivation of ATB as an appropriate behavioral assumption especially if own effects on the aggregate are negligible, e.g. in large games. In case of the sum-aggregative games considered here, the difference in behavior manifests itself in the respective FOC's and the corresponding slopes. Consider a sum-aggregative game with strongly quasiconcave payoffs ${ }^{8} \Pi^{j}\left(x_{j}, Q\right)$ in $x_{j}$ for any given $Q=\sum_{i} x_{i}$, and only interior solutions of the respective optimization problems, i.e. $\varphi_{A}^{j}(Q) \in \operatorname{Int}(\mathcal{S})$. Then, the ATB joint-response corresponds to the function $\phi_{A}(x)=\left(\varphi_{A}^{j}(Q)\right)_{j=1}^{N}$, with slopes $\partial \varphi_{A}^{j}(Q) \equiv b_{A}^{j}(Q)=-\frac{\Pi_{12}^{j}}{\Pi_{11}^{j}}$. An interior ATB equilibrium $x_{A}^{*}$ solves $\phi_{A}\left(x_{A}^{*}\right)=x_{A}^{*}$. Under ATB the best-response dynamics are described by the recurrence relation $x^{t+1}=\phi_{A}\left(x^{t}\right)=\left(\varphi_{A}^{j}\left(\sum x_{i}^{t}\right)\right)_{j=1}^{N}$. Hence we can use the same machinery as before to assess the local contraction-stability of an ATB-equilibrium by verifying whether or not $\phi_{A}$ is a local contraction at $x_{A}^{*}$ :

Proposition 4 Suppose that $x_{A}^{*}$ is an interior ATB equilibrium with aggregate $Q^{*}$. Then $x_{A}^{*}$ is contraction-stable under the ATB response dynamics if and only if $\left|\sum_{j=1}^{N} b_{A}^{j}\left(Q^{*}\right)\right|<1$. Moreover, if the previous condition holds for any $Q(x)=\sum x_{i}$ with $x \in \operatorname{Int}(\mathcal{S})$, the game has a unique and globally contraction-stable ATB equilibrium.

\footnotetext{
${ }^{8} \Pi_{1}^{j}\left(x_{j}, Q\right)=0 \Rightarrow \Pi_{11}^{j}\left(x_{j}, Q\right)<0$
} 
Proof:

The first claim can be proved by using corollary 2 and lemma 1, noting that the $j$-th row of the matrix $\partial \phi_{A}(x)$ has the form $\left(b_{A}^{j}, \ldots, b_{A}^{j}\right)$, i.e. an EV $\lambda$ of $\partial \phi_{A}(x)$ either is zero or solves $\lambda=\sum b_{A}^{j}$. Alternatively, we can sum up the equations $\varphi_{A}^{j}(Q)=x_{j}$ to obtain the single equilibrium equation $\sum \varphi_{A}^{i}(Q)=Q$. Hence $Q^{*}$ is contraction-stable if and only if $\left|\frac{\partial \sum \varphi_{A}^{i}\left(Q^{*}\right)}{\partial Q}\right|<1$, which also proves the first claim. The second claim follows from corollary 1 and the Banach FP theorem.

Let $\nabla \Pi_{A}(x) \equiv\left(\nabla_{j} \Pi^{j}\left(x_{j}, Q\right)\right)_{j=1}^{N}$ be the $N$-vector obtained by stacking all players ATB gradients (remember that players treat $Q$ as fixed), with corresponding Jacobian $H_{A}(x)=\frac{\partial \nabla \Pi_{A}(x)}{\partial x}$. Any candidate for an interior ATB equilibrium belongs to $C r_{A} \equiv\left\{x \in \operatorname{Int}(\mathcal{S}): \nabla \Pi_{A}(x)=0\right\}$. I call a game with $\mathrm{ATB}, \phi_{A}(\mathcal{S}) \subset \operatorname{Int}(\mathcal{S})$ and $\operatorname{Det}\left(H_{A}(x)\right) \neq 0$ on $C r_{A}$ an ATB index game. As mentioned above, ATB has the advantage of simplifying the involved algebra. This can be seen in the proof of the next theorem, which states that with ATB contraction-stability at critical points is always sufficient for uniqueness, and necessary under complements (i.e. if $b_{j}^{A} \geq 0$ on $\left.C r_{A}\right)$.

Theorem 5 A sum-aggregative ATB index game has a unique equilibrium if and only if $\sum b_{A}^{j}(Q(x))<1$ on $C r_{A}$. If every $x \in C r_{A}$ is $A T B$ contraction-stable, then there is a unique ATB equilibrium. Moreover, if $b_{A}^{j}(x) \geq 0 \forall j$ on $C r_{A}$, then the game has a unique ATB equilibrium if and only if every $x \in C r_{A}$ is ATB contraction-stable.

Proof:

Suppose that $x \in C r_{A}$. Note that the $j$-th row of $H_{A}(x)$ has all entries equal to $\Pi_{12}^{j}$ except the diagonal entry, which is $\Pi_{11}^{j}+\Pi_{12}^{j}$. Using lemma 1 we immediately see that $\operatorname{Det}\left(-H_{A}(x)\right)>0$ if and only if $\prod_{j}\left(-\Pi_{11}^{j}\right)\left(1-\sum_{i} b_{i}^{A}\right)>0$. Consequently, there is a unique ATB equilibrium if and only if $\sum_{i} b_{i}^{A}(Q(x))<1$, where $Q(x)=\sum x_{i}$ by the index theorem. All remaining claims then follow from proposition 4 .

This result is similar to theorem 4, but a glance at the proof shows that it requires considerably less effort to be established. Moreover it is notable, that theorem 5 does not generally require the slopes to have the same signs at critical points. This originates from the convenient property 
of ATB that every row of $\partial \phi_{A}(x)$ has the same entries, which implies that there is at most one non-zero EV $\lambda$ of $\left(\partial \phi_{A}(x)\right)$ independent of the signs of the entries, and $\rho\left(\partial \phi_{A}(x)\right)=|\lambda|$.

Does ATB stabilize or destabilize a given game compared to the NB with respect to the induced best-response dynamics? A direct comparison of proposition 4 to theorem 3 suggests that, unfortunately, the question may be hard to answer generally, as usually $x_{A}^{*} \neq x^{*}$. Nevertheless, section 4.3 shows with linear and symmetric best-responses there is a clear-cut result, supported by a nice intuition.

\section{Symmetric games}

Besides the class of sum-aggregative games, the class of symmetric games is another candidate where we can expect to uncover some pattern between uniqueness and contraction-stability due to the symmetric structure of the best-replies. Symmetric games have been of considerable importance in applied work (e.g. Salop (1979), Grossman and Shapiro (1984), Hefti (2011)), mainly because symmetry simplifies the analysis. In this section I apply previous results to symmetric games. I provide a simple characterization for the local contraction-stability of a symmetric equilibrium and, similar to section 3 , relate stability to uniqueness. As before, there is a crucial difference if strategies are either substitutes or complements. As symmetric games are highly tractable, contraction-stability can be effectively compared to (time-continuous) gradientstability. Whereas in case of complements these two dynamical concepts are equivalent, gradientstability is less restrictive in terms of the slope coefficients with substitutes. However, this difference evaporates if players follow a sequential rather than a simultaneous updating process. As illustrated in a simple example, these findings can be of economic interest e.g. if individual adjustments change over time and a presumably stable equilibrium bifurcates to an unstable one without changing the conventional parameters of the game. 


\subsection{Uniqueness and contraction-stability}

Let $M$ denote a partitioned $N k \times N k$-matrix with $N \times N$ partitions of the form

$$
M=\left(\begin{array}{ccccc}
0 & A & A & \cdots & A \\
A & 0 & A & \cdots & A \\
\cdots & \cdots & \cdots & \cdots & \cdots \\
\cdots & \cdots & \cdots & \cdots & \cdots \\
A & A & \cdots & A & 0
\end{array}\right)
$$

Lemma 2 The spectral radius of $M$ is $\rho(M)=(N-1) \rho(A)$.

Proof: Section 6.6

Note that for $k=1$ the result follows from lemma 1. It is not hard to see that in a symmetric $k$-dimensional $N$-player game the matrix $\partial \phi\left(x^{*}\right)$ takes on the form (4) at a symmetric equilibrium $x^{*}$, where 0 and $A$ both are $k \times k$ matrices and $A=\frac{\partial \varphi^{j}\left(x^{*}\right)}{\partial x_{g}}, g \neq j$. From Lemma 2 and corollary 2 we immediately get the following characterization of contraction-stability at symmetric equilibria:

Theorem 6 The interior symmetric equilibrium $x^{*}$ is contraction-stable if and only if $(N-$ 1) $\rho\left(\frac{\partial \varphi^{1}\left(x^{*}\right)}{\partial x_{2}}\right)<1$.

Hence to check for contraction-stability we only need to evaluate the slopes of the best-response function of an arbitrary player with respect to the strategy of some other player. Let $\tilde{\varphi}(\bar{x}) \equiv$ $\varphi^{1}(\bar{x}, \ldots, \bar{x}), \bar{x} \in S$, and note that $x^{*}=\left(x_{1}^{*}, \ldots, x_{1}^{*}\right)$ is an interior symmetric equilibrium if and only if $\tilde{\varphi}\left(x_{1}^{*}\right)=x_{1}^{*}$. The advantage of this formulation especially in applications is that we only need to write down the profit function as $\Pi\left(x_{1}, \bar{x}, \ldots, \bar{x}\right)$ and solve the respective FOC at symmetric points. Theorem 6 can be succinctly restated in terms of $\tilde{\varphi}$ :

Corollary 7 The interior symmetric equilibrium $x^{*}$ is contraction-stable if and only if $\rho\left(\frac{\partial \tilde{\varphi}\left(x_{1}^{*}\right)}{\partial \bar{x}}\right)<$ 1.

\section{Proof:}

Follows from theorem 6 and $\frac{\partial \tilde{\varphi}\left(x_{1}^{*}\right)}{\partial \bar{x}}=(N-1) \frac{\partial \varphi^{1}\left(x^{*}\right)}{\partial x_{h}}$. 
See the proof of proposition 5 for how $\frac{\partial \tilde{\varphi}\left(x_{1}^{*}\right)}{\partial \bar{x}}$ can be expressed in terms of partial derivatives of $\Pi$. In now turn to the relationship between uniqueness and contraction-stability in symmetric games. Any equilibrium in a symmetric games must either be symmetric or asymmetric, and if $x^{*}$ is an asymmetric equilibrium, there must be at least $N-1$ further asymmetric equilibria. Under fairly general conditions, a symmetric game possesses at least one symmetric equilibrium (Hefti (2011)). Hence uniqueness may fail to hold in symmetric games because there are asymmetric equilibria or multiple symmetric equilibria. Let us first deal with the relationship between contraction-stability and multiplicity of symmetric equilibria. Let $\nabla_{1} \tilde{\Pi}^{1}\left(x_{1}\right) \equiv \nabla_{1} \Pi^{1}\left(x_{1}, \ldots, x_{1}\right)$. Hence $\nabla_{1} \tilde{\Pi}^{1}: S(k) \rightarrow \mathbb{R}^{k}$ defines a vector field over $S(k)$ with corresponding Jacobian $\tilde{H}\left(x_{1}\right) \equiv$ $\frac{\partial \nabla_{1} \tilde{\Pi}^{1}\left(x_{1}\right)}{x_{1}}$. Note that any interior symmetric equilibrium $x^{*}=\left(x_{1}^{*}, \ldots, x_{1}^{*}\right)$ has $x_{1}^{*} \in C r^{s} \equiv$ $\left\{x_{1} \in \operatorname{Int}(S(k)): \nabla_{1} \tilde{\Pi}^{1}\left(x_{1}\right)=0\right\}$. I call a symmetric game, where $\nabla_{1} \tilde{\Pi}^{1}$ points into $S(k)$ at boundary points ${ }^{9}$ and $\operatorname{Det}\left(\tilde{H}\left(x_{1}\right)\right) \neq 0$ on $C r^{s}$ a symmetric index game. For such an index game we can use the index theorem to count the multiplicity of symmetric equilibria (their number must always be odd, see Hefti (2011) for details). Moreover, a symmetric index games has exactly one symmetric equilibrium if and only if $\operatorname{Det}\left(-\tilde{H}\left(x_{1}\right)\right)>0$ on $C r^{s}$. The following proposition says that in general (i.e. for $k \geq 1$ ) local contraction-stability implies the existence of exactly one symmetric equilibrium.

Proposition 5 Consider a $k$-dimensional symmetric index game. If every $x_{1} \in C r^{s}$ is contractionstable, then there is exactly one symmetric equilibrium.

Proof:

Suppose that $x_{1} \in C r^{s}$ and let $A\left(x_{1}\right)=\frac{\partial^{2} \Pi^{1}\left(x_{1}, x_{-1}\right)}{\partial x_{1} \partial x_{1}}$ and $B\left(x_{1}\right)=\frac{\partial^{2} \Pi^{1}\left(x_{1}, x_{-1}\right)}{\partial x_{1} \partial x_{2}}(N-1)$, both evaluated at $\left(x_{1}, \ldots, x_{1}\right)$. The IFT implies that $\frac{\partial \tilde{\varphi}\left(x_{1}\right)}{\partial \bar{x}}=-\left(A\left(x_{1}\right)\right)^{-1} B\left(x_{1}\right)$. Next, note that $\tilde{H}\left(x_{1}\right)$ can be written as $\tilde{H}\left(x_{1}\right)=A\left(x_{1}\right)+B\left(x_{1}\right)$ so simple algebra shows that $\operatorname{Det}\left(-\tilde{H}\left(x_{1}\right)\right)>0$ if and only if $\operatorname{Det}\left(I-\frac{\partial \tilde{\varphi}\left(x_{1}\right)}{\partial \bar{x}}\right)>0$. Now, $\lambda \in \sigma\left(\frac{\partial \tilde{\varphi}\left(x_{1}\right)}{\partial \bar{x}}\right)$ if and only if $(1-\lambda) \in \sigma\left(I-\frac{\partial \tilde{\varphi}\left(x_{1}\right)}{\partial \bar{x}}\right)$. Consequently, if $x^{*}$ is contraction-stable, we must have $|\lambda|<1$ for any $\mathrm{EV}$ of $\frac{\partial \tilde{\varphi}\left(x_{1}\right)}{\partial \bar{x}}$, which in turn implies $\operatorname{Det}\left(I-\frac{\partial \tilde{\varphi}\left(x_{1}\right)}{\partial \bar{x}}\right)=\prod_{i=1}^{k}\left(1-\lambda_{i}\right)>0$ as can easily be verified, and the claim follows from the index theorem.

\footnotetext{
${ }^{9}$ For $k=1$ this is equivalent to saying that the game has no symmetric boundary equilibria.
} 
Put differently, proposition 5 says that whenever a symmetric index game has multiple symmetric equilibria, there must be unstable equilibria. We also see that the equivalence between uniqueness and local contraction-stability encountered in section 3.1 in case of equilibrium complements appears to be a one-dimensional phenomenon, as $\operatorname{Det}\left(-\tilde{H}\left(x_{1}\right)\right)>0$ does not necessarily imply $\rho\left(\frac{\partial \tilde{\varphi}\left(x_{1}\right)}{\partial \bar{x}}\right)<1$ even if $\frac{\partial \tilde{\varphi}\left(x_{1}\right)}{\partial \bar{x}}>0$ already for $k=2$. But if $k=1$ we get a similar result concerning uniqueness and stability as in section 3.1. First, note that the equivalence between contraction-stability and local dominance solvability necessarily extends to symmetric equilibria and leads to the following characterization of contraction-stability of symmetric equilibria in one-dimensional symmetric games:

Proposition 6 Let $x^{*}$ be an interior symmetric equilibrium of a one-dimensional symmetric game. Then $x^{*}$ is contraction-stable if and only if one of the following conditions is satisfied:
1) $\left|\frac{\partial \tilde{\varphi}\left(x_{1}\right)}{\partial \bar{x}}\right|<1$
2) $R_{m}\left(x^{*}\right)<1 \forall m$
3) $H\left(x^{*}\right)$ has a dominant diagonal
4) The game is locally dominance solvable at $x^{*}$

Proof:

The first two equivalence relations follow from corollary 7 as $\rho\left(\frac{\partial \varphi^{1}\left(x^{*}\right)}{\partial x_{2}}\right)=\left|\frac{\partial \varphi^{1}\left(x^{*}\right)}{\partial x_{2}}\right|(N-1)=$ $\left|\frac{\partial \tilde{\varphi}\left(x_{1}\right)}{\partial \bar{x}}\right|$, and 2) $\left.\Leftrightarrow 3\right)$ follows from the IFT. The last equivalence follows from Moulin's result mentioned earlier.

Notably, diagonal dominance of the Jacobian $H\left(x^{*}\right)$ is not only sufficient for contraction-stability (which we already know from section 2.4) but also necessary ${ }^{10}$ in case of a symmetric game. The following proposition shows that the inexistence of multiple symmetric equilibria and contraction-stability are the same properties of a game where strategies are complements at symmetric equilibria. Further, these properties are independent of whether or not there are asymmetric equilibria. In Hefti (2011) it is shown that

$$
\Pi_{1}\left(x_{1}, x_{2} ; X\right)=0 \Longrightarrow-\frac{\Pi_{12}\left(x_{1}, x_{2} ; X\right)}{\Pi_{11}\left(x_{1}, x_{2} ; X\right)}>-1 \quad, X \equiv\left(x_{3}, \ldots, x_{N}\right)
$$

\footnotetext{
${ }^{10}$ For $k>1$ this equivalence generally breaks down (but because of corollary 5 the sufficiency part still holds).
} 
is a sufficient condition for the inexistence of asymmetric equilibria.

Proposition 7 Let $x^{*}$ be an interior symmetric equilibrium of a one-dimensional symmetric index game.

1) If $\frac{\partial \tilde{\varphi}\left(x_{1}\right)}{\partial \bar{x}} \geq 0$ on $C r^{s}$, then $x^{*}$ is the only symmetric equilibrium if and only if any $x_{1} \in C r^{s}$ is contraction-stable.

2) If $\frac{\partial \tilde{\varphi}\left(x_{1}\right)}{\partial \bar{x}} \leq 0$ on $C r^{s}, x^{*}$ is the only symmetric equilibrium, and if $(N-1) \Pi_{12}\left(x_{1}, x_{-1}\right)>$ $\Pi_{11}\left(x_{1}, x_{-1}\right)$ holds for any $x_{-1} \in S^{N-1}$ whenever $\Pi_{1}\left(x_{1}, x_{-1}\right)=0$, then $x^{*}$ is both unique and contraction-stable.

Proof:

The proof of proposition 5 reveals that there is exactly one symmetric equilibrium if and only if $\frac{\partial \tilde{\varphi}\left(x_{1}\right)}{\partial \bar{x}}<1$ on $C r^{s}$, and the first claim follows from proposition 6. Similarly, if $\frac{\partial \tilde{\varphi}\left(x_{1}\right)}{\partial \bar{x}} \leq 0$ on $C r^{s}$, there is exactly one symmetric equilibrium. The remaining claim is true, as $(N-$ 1) $\Pi_{12}\left(x_{1}, x_{-1}\right)>\Pi_{11}\left(x_{1}, x_{-1}\right)$ on $\Pi_{1}\left(x_{1}, x_{-1}\right)=0$ implies both contraction-stability of any symmetric equilibrium candidate and condition (5) to hold.

Obviously, in case of equilibrium symmetric complements there can be a single symmetric equilibrium (necessarily contraction-stable) with our without the existence asymmetric equilibria. However, if additionally (5) is satisfied, then the symmetric equilibrium necessarily is unique. Summarizing, we note that a similar divergence as encountered in section 3.1 between uniqueness and stability in dependence of the strategy type is also present in symmetric games. Further, proposition 7 shows that with substitutes the symmetric equilibrium is a contraction if the replyfunctions are sufficiently flat ${ }^{11}$, which in turn limits the scope of asymmetric equilibria. In case of complements a change in the number of players affects both the scope for multiple symmetric equilibria and whether a symmetric equilibrium is contraction-stable. Such a relation does not hold for games with substitutes. Below it is shown that the restrictive nature of contractionstability in case of symmetric substitutes crucially originates from the requirement that all players update their behavior simultaneously.

\footnotetext{
${ }^{11}$ It is noteworthy that for $N>2$ the above condition generally is weaker than diagonal dominance.
} 


\subsection{Gradient dynamics, sequential adjustments and uniqueness}

The notion of contraction-stability implicitly entails the simultaneity of the best-response adjustments in its definition. What happens if players adjust sequentially, e.g. by taking turns? Although this question has been raised earlier (Gabay and Moulin (1980) and Moulin (1984)), there has been no clear-cut answer, as examples can be constructed, where an equilibrium is stable under sequential adjustments but not under simultaneous adjustments and vice-versa. This section illustrates that in symmetric games a stability ranking of the two different adjustment processes exists in terms of the restrictions imposed on the best-response slope coefficient, and compares these restrictions to those imposed by gradient dynamics.

I refer to the time-continuous adjustment process $x(t)$ induced by the $N$ FOCs, $\dot{x}_{j}=s \Pi_{1}^{j}(x)$ for $1 \leq j \leq N$, as gradient dynamics (see e.g. Dixit (1986), Vives (1999), Dastidar (2000) or Hefti (2011) for applications). $s>0$ is an arbitrary speed of adjustment. Let $\hat{H}(x)$ denote the Jacobian associated with the right sides of these equations. An interior equilibrium $x^{*}$ is (locally) gradient stable if all eigenvalues of $\hat{H}(x)$ have negative real parts.

Lemma 3 Suppose $x^{*}$ is a symmetric interior equilibrium. Then $x^{*}$ is gradient stable if and only if

$$
\frac{\partial \tilde{\varphi}\left(x_{1}^{*}\right)}{\partial \bar{x}} \in(-(N-1), 1) \quad \text { or } \quad \frac{\partial \varphi^{1}\left(x_{1}^{*}\right)}{\partial x_{2}} \in\left(-1, \frac{1}{N-1}\right)
$$

Proof:

As $\hat{H}\left(x^{*}\right)=s H\left(x^{*}\right)$ is a symmetric matrix, all eigenvalues are negative if and only if $-H\left(x^{*}\right)$ is positive definite. Using lemma 1 and $\frac{\partial \hat{\varphi}\left(x_{1}^{*}\right)}{\partial \bar{x}}=-\frac{(N-1) \Pi_{12}^{1}\left(x^{*}\right)}{\Pi_{11}^{1}\left(x^{*}\right)}=(N-1) \frac{\partial \varphi^{1}\left(x_{1}^{*}\right)}{\partial x_{2}}$ it easily is verified that this is the case if and only if condition (6) holds.

While the slope domain of $\frac{\partial \tilde{\varphi}\left(x_{1}^{*}\right)}{\partial \bar{x}}$ asserting contraction-stability is symmetric around 0 , this is not the case for the gradient dynamics (if $N>2$ ). Nevertheless, we see that gradient-stability, contraction-stability, local dominance-solvability and the existence of exactly one symmetric equilibrium are the same formal properties of a symmetric index game with equilibrium complements. Hence under complements very different dynamic processes (in case of dominancesolvability infinite foresight) all make the same stability prediction. As a remark it should also be noted that by the open mapping principle these results stay valid under sufficiently small (asymmetric) perturbations of the exogenous parameters of the game. The asymmetry in condition 
(6) reveals an interesting tension between the response to a change of an individual strategy and to a change of all the opponents' strategies. With complements the individual slope condition is always satisfied but the collective slope conditions might be violated whereas the opposite holds with substitutes.

I now illustrate that the relative restriction imposed by contraction-stability compared to the gradient dynamics originate from the simultaneity entailed in its definition. For a given ordering $\{1, \ldots, N\}$ of the players the sequential dynamic is defined by the sequence $\left(x^{t}\right)$ with components evolving according to:

$$
x_{i}^{t+1}= \begin{cases}\varphi^{i}\left(x_{-i}^{t}\right) & \frac{1+t-i}{N} \in \mathbb{N} \\ x_{i}^{t} & \text { else }\end{cases}
$$

I call the symmetric equilibrium $x^{*}$ sequentially stable if $x^{t} \rightarrow x^{*}$ locally, and convergence occurs if (7) induces a local contraction. In order to establish $x^{t} \rightarrow x^{*}$ it suffices to show that the subsequence $x^{N}, x^{2 N}, x^{3 N}, \ldots$ converges to $x^{*}$. Let $y^{t}=x^{t N}$ and consider the sequence $y^{t+1}=z\left(y^{t}\right)$, where $z\left(y^{t}\right)=z_{N} \circ z_{N-1} \circ \ldots \circ z_{1}\left(y^{t}\right)$ with $z_{j}(y)=\left(y_{1}, \ldots, \varphi^{j}\left(y_{-j}\right), \ldots, y_{N}\right)$. By corollary 3 the composite mapping $z$ is a local contraction at $x^{*}$ if and only if

$$
\begin{aligned}
L(x)=z\left(x^{*}\right)+\partial z\left(x^{*}\right) \cdot\left(x-x^{*}\right) & =x^{*}+\partial z_{N}\left(x^{*}\right) \cdot \ldots \cdot \partial z_{1}\left(x^{*}\right) \cdot\left(x-x^{*}\right) \\
& =x^{*}+A\left(x^{*}\right) \cdot\left(x-x^{*}\right)
\end{aligned}
$$

is a contraction, i.e. if and only if $\rho\left(A\left(x^{*}\right)\right)<1$. Hence, if $\rho\left(A\left(x^{*}\right)\right)<1$ the sequence $\left(y^{t}\right)$ converges (locally) to $x^{*}$. By symmetry, $\partial z_{j}\left(x^{*}\right)$ can be represented by the $N \times N$-matrix $\partial z_{j}=\left(z_{l m}\right)$ with

$$
z_{l m}= \begin{cases}1 & l=m \neq j \\ a & l=j \neq m \\ 0 & \text { else }\end{cases}
$$

and $a=\frac{\partial \varphi^{1}\left(x^{*}\right)}{\partial x_{2}}$. Then, $A\left(x^{*}\right)=\prod_{j=1}^{N} \partial z_{j}\left(x^{*}\right)$ is a $N \times N$-matrix, where the first column has only zeroes as entries. Hence the non-trivial eigenvalues $\lambda$ of $A\left(x^{*}\right)$ can be found by solving $\hat{A}\left(x^{*}\right) v=\lambda v$, where $\hat{A}$ is obtained from $A$ by deleting the first row and column. For $N=4$ we 
obtain

$$
\hat{A}\left(x^{*}\right)=\left(\begin{array}{ccc}
a^{2} & a(1+a) & a(1+a) \\
a^{2}(1+a) & a^{2}(2+a) & a(1+a)^{2} \\
a^{2}(1+a)^{2} & a^{2}(1+a)(2+a) & a^{2}(3+a(3+a))
\end{array}\right)
$$

Note that the submatrix obtained by deleting the last row and column of $\hat{A}$ corresponds to the situation if there were $N=3$ players, and deleting the last two rows and columns leads to the case of $N=2$ players. If $a \geq 0$ and we use the maximum row-sum norm to bound the spectral radius, we obtain that $\rho(\hat{A})<1$ if $a<1 /(N-1)$ for $N=2,3,4$. For $a<0$ using the same norm reveals that $\rho(\hat{A})<1$ if $a>-1$, for $N=2,3,4$. These simple calculations show that if $a \in\left(-1, \frac{1}{N-1}\right), N \in\{2,3,4\}$, then $\rho\left(A\left(x^{*}\right)\right)<1$, i.e. sequential stability results. Comparing this to 3 we see that the stability domain of the sequential dynamics corresponds at least to the domain of the gradient dynamics. In fact, by explicitly calculating the spectral radius it can be shown that this bound is sharp, i.e. the two domains coincide. Unfortunately, the question how $\rho\left(A\left(x^{*}\right)\right)$ depends on $a$ cannot be answered analytically for general $N$ in a simple way, as the maximum row-sum norm (or other conventional norms) do not efficiently bound the spectral radius if $N>4$ and $a<0$. Using numerics ${ }^{12}$ to calculate the eigenvalues in dependence of $(a, N)$ reveals the stability regions of the sequential dynamics and the gradient dynamics to coincide also for $N>4$ (left picture of figure 1). These findings can be summarised as:
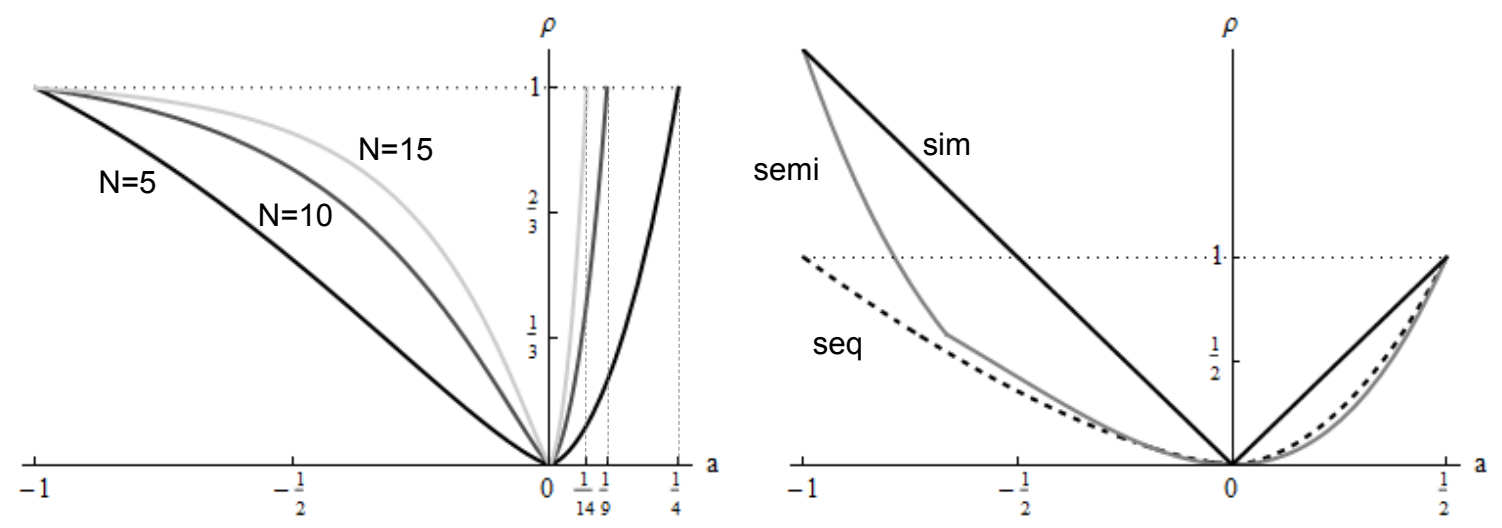

Figure 1: Spectral radius

Observation 1 For symmetric equilibria the stability regions of the sequential and the gradient dynamics coincide.

\footnotetext{
${ }^{12}$ The numerical simulation was conducted on a Mathematica 8.0 platform.
} 
The difference between the simultaneous and the sequential dynamics with substitutes is interesting. It means that an equilibrium, which is unstable under simultaneous updating, might be stable if the players only update infrequently, e.g. because they allocate their limited attention among several different activities. While small shocks would be absorbed by the sequential dynamics, this may not hold for the simultaneous dynamics. Further numerical examination support the conjecture of the dynamics gradually approaching the simultaneous dynamics in case of substitutes the more frequently the players update. This is illustrated in the right picture of figure 1 for $N=3$, where "sim" is the simultaneous and "seq" the sequential dynamics. "Semi" corresponds to a case, where players 2 and 3 behave sequentially but player 1 updates every period. Summarizing, we record that if players start to concentrate more on a symmetric game with substitutes, i.e. they update their responses more frequently, a previously stable pattern can become unstable. It would certainly be of interest to test such a conjecture in the lab.

If the symmetric index game under consideration is of a sum-aggregative nature and e.g. costs are strictly convex, the following strong result can be obtained:

Proposition 8 A sum-aggregative symmetric index game with equilibrium substitutes and $\Pi_{11}\left(x_{1}, \sum x_{i}\right)<0$ has a unique and gradient (or sequentially) stable symmetric equilibrium.

Proof:

Equilibrium substitutes, lemma 3 and observation 1 imply that the only symmetric equilibrium $x^{*}$ is gradient (or sequentially) stable if and only if $\frac{\Pi_{12}\left(x^{*}\right)}{\Pi_{11}\left(x^{*}\right)}<1$. Hence, if (5) is satisfied, there cannot be any asymmetric equilibria and $x^{*}$ must be gradient stable. But for sum-aggregative games condition (5) is equivalent to $\Pi_{11}\left(x_{1}, \sum x_{i}\right)<0$.

Both the Cournot-game and the rent-seeking game mentioned in section 3 are prone to equilibrium substitutes. If in the Cournot example the strategies (quantities) are substitutes $\left(P^{\prime}+\right.$ $\left.x_{1} P^{\prime \prime}<0\right)$ and costs are strictly convex, then proposition 8 implies that the symmetric equilibrium is the unique and gradient stable equilibrium. ${ }^{13}$ Similarly, the rent-seeking game $\Pi^{1}=$ $\pi\left(\frac{x_{1}}{\sum x_{j}+r}\right)-c\left(x_{1}\right)$, with $\pi^{\prime}>0, r, c^{\prime \prime} \geq 0$ and $\pi^{\prime \prime} \leq 0$, with equilibrium substitutes $\left(-\frac{\pi^{\prime \prime}(z) z}{\pi^{\prime}(z)}<1\right)$

\footnotetext{
${ }^{13}$ This generalizes a result of Dastidar (2000) to arbitrary sum-aggregative games with substitutes.
} 
has a unique and gradient (or sequentially) stable symmetric equilibrium. Further, in both examples the symmetric equilibrium usually is prone to violate (simultaneous) contraction-stable if $N>2$. This can be illustrated by considering the class of homogeneous symmetric games. Suppose that $\Pi\left(x_{j}, x_{-j}\right)=\pi\left(x_{j}, x_{-j}\right)-\theta x_{j}^{\eta}$, where $\pi$ is homogeneous of some degree $k$ in $\left(x_{j}, x_{-j}\right)$ and $\eta \geq 1$. The Tullock contest success function, $\pi^{j}=\left(\sum_{n} x_{j}\right)^{r} V$, where $r \in(0,1]$, is an important example for $k=0$ (as only relative efforts matter for success). The Cournot model with iso-elastic market demand, $\pi^{j}=\left(\sum_{n} x_{n}\right)^{k-1} x_{j}$ is an example where homogeneity degree $k \in[0,1)$ is possible. Let $\varepsilon=(N-1) x_{1} \frac{\partial^{2} \pi}{\partial x_{1} \partial x_{2}} / \frac{\partial \pi}{\partial x_{1}}$.

Proposition 9 For any $k<1$ there exists exactly one symmetric equilibrium $x^{*}$, which is contraction-stable (or locally dominance-solvable) if and only if $\rho=\left|\frac{\varepsilon}{(\eta-k)+\varepsilon}\right|<1$.

Proof:

As $\tilde{\pi}_{1}\left(x_{1}\right) \equiv \pi_{1}\left(x_{1}, x_{1}, \ldots, x_{1}\right)$ is $(k-1)$-homogeneous in $x_{1}, \tilde{\pi}_{1}\left(x_{1}\right)=\tau x_{1}^{k-1}, \tau>0$, which shows that $\tilde{\pi}_{1}(x)-\theta \eta x^{\eta-1}=0$ has a unique solution whenever $k<1$. Using the IFT and $(k-1)$ homogeneity of $\pi_{1}$ gives $\frac{\partial \tilde{\varphi}\left(x_{1}\right)}{\partial \bar{x}}=\frac{\varepsilon}{(\eta-k)+\varepsilon}$ in any symmetric equilibrium, and the claim follows from proposition 6 .

The following table depicts that, unless $\eta>1$, the symmetric equilibrium usually is not contraction-stable.

\begin{tabular}{|c|c|c|c|c|}
\hline Game & Parameter & $\begin{array}{c}\text { Complements } \\
\text { iff }\end{array}$ & $\begin{array}{c}\text { Contraction- } \\
\text { stability }\end{array}$ & $\begin{array}{c}\text { Gradient } \\
\text { stability }\end{array}$ \\
\hline Tullock & $r \in(0,1]$ & $r \leq \frac{1}{N-1}$ & $r<\frac{2}{N-1}$ & $r \in(0,1]$ \\
\hline Cournot & $k \in[0,1)$ & $k=0, N=2$ & $N=2$ & $k \in[0,1)$ \\
\hline
\end{tabular}

Table 1: Stability regions for $\eta=1$

As $\varepsilon$ is zero-homogeneous in $x$, so is $\frac{\partial \tilde{\varphi}\left(x_{1}\right)}{\partial \bar{x}}$, which further implies that $\varepsilon\left(x_{1}, \ldots, x_{1}\right)$ must be constant. Hence $\rho^{\prime}(\eta)<0$ unambiguously. ${ }^{14}$ Further, proposition 9 suggests that with substitutes a lower value of $k$ tends towards making the equilibrium more likely to be stable. Formally, $\rho^{\prime}(k)>0$ holds if and only if $\varepsilon(k)>(k-\eta) \varepsilon^{\prime}(k)$, which may or may not be satisfied in the Cournot-example (but mostly it is satisfied), depending on the exact parameter constellation.

\footnotetext{
${ }^{14}$ It should be noted that while this statement holds in homogeneous games, it can be violated as $x^{*}$ also depends on parameters influencing the elasticity of the costs.
} 
Summarizing, we observe that while we cannot expect simultaneous contraction-stability to occur if strategies are substitutes in the Tullock or Cournot-example unless costs are sufficiently convex, sequential stability (or gradient stability) is necessarily satisfied at the unique symmetric equilibrium of the game.

\subsection{Linear sum-aggregative games and ATB}

In this section I compare the ATB dynamics to the NB dynamics in terms of their stability in case of a symmetric linear sum-aggregative game. Let $Q=\sum_{i} x_{i}$ and $\bar{Q}_{j}=\sum_{i \neq j} x_{i}$. Under NB the players optimize $\Pi^{j}\left(x_{j}, x_{j}+\bar{Q}_{j}\right)$ for given $\bar{Q}_{j}$, whereas under ATB the optimize $\Pi^{j}\left(x_{j}, Q\right)$ for given $Q$. The FOC of the NB-problem is linear in $\left(x_{j}, \bar{Q}_{j}\right)$ if and only if the FOC of the ATB-problem is linear in $\left(x_{j}, Q\right)$. Hence, without loss of generality, we can work with $\Pi^{j}=A x_{j}+B Q x_{j}-\frac{1}{2} c x_{j}^{2}$. As we are interested only in stability we can choose $A=0$ and set $c>0$ and $2 B<c$. Then $x_{A}^{*}=x^{*}=0, b_{j}=\frac{B}{c-2 B}$ and $b_{A}^{j}=\frac{B}{c}$.

Proposition 10 If strategies are complements $(B \geq 0)$ then the equilibrium is ATB contractionstable whenever it is NB contraction-stable. Under substitutes the opposite direction applies.

Proof:

Let $\rho \equiv \rho(\partial \phi)$ and $\rho_{A} \equiv \rho\left(\partial \phi_{A}\right)$. From the proof of proposition 4 we obtain $\rho_{A}=\left|\sum b_{A}^{j}\right|=\frac{N|B|}{c}$. Further $\rho=\frac{(N-1)|B|}{(c-2 B)}$ follows from lemma 2. If $B \geq 0$, then plugging $\frac{(N-1) B}{c-2 B}=\varepsilon$ into $\rho_{A}$ gives $\frac{N \varepsilon}{(N-1)+2 \varepsilon}$. Hence $\rho_{A}<1$ if and only if $N \varepsilon<(N-1)+2 \varepsilon$, which holds whenever $\varepsilon<1$, proving the first claim. The second claim follows as $\rho_{A}=-\frac{B N}{c}>-\frac{B N}{c-2 B}>\rho$.

There are two differences between ATB and NB relevant for stability. First, the ATB dynamics have an additional potential for instability originating from the fact that a player is updating his strategy over his own prior action, not just over the actions of his opponent. Formally, this manifests itself in the fact that the response matrix $\partial \phi_{A}$ may have a non-zero diagonal. If all slopes under ATB and under NB were identical (which is never possible in a linear game unless $B=0)$ then $\rho_{A} \geq \rho$. The second effect originates from the fact that an NB-player takes into account his own effect on the aggregate when updating his response, while an ATB-player does not. To illustrate, consider the decision of player 1 and suppose that $d x_{2}>0$. Then $d \bar{Q}_{1}>0$, 
which under complementarity implies $d x_{1}>0$ for both ATB and NB. But $d x_{1}>0$ also means $d Q>0$, which is taken into account only by the NB-player and, by complementarity, induces him to increase $x_{1}$ even further. Thus for complements player 1's response towards a change of an opponent's strategy is stronger under NB than under ATB, which manifests itself in a steeper slope of his response function. But steeper slopes, i.e. stronger individual responses, always tend towards making the NB dynamics less stable. If strategies are substitutes the direction is reversed by the same logic, i.e. the fact that the players take into account their own effects on the aggregate flattens their response and tends towards stabilizing NB.

The presence of the above two potentially countervailing effects explains why in general we cannot expect to find a clear-cut stability ranking between NB and ATB. Nevertheless, proposition 10 shows that with linear response functions the second (player-specific) effect unambiguously is the dominant one.

As a final remark it is a general consequence of theorem 2 (not restricted to symmetric games) that if $Q(x)$ is a function continuous at $x^{*}$ (e.g. an average of some strategies), sequences of the type $Q^{t}=Q\left(x^{t}\right)$ converge (locally) to $Q\left(x^{*}\right)$ if $x^{t} \rightarrow x^{*}$. In general, the converse is false (e.g. consider $Q(x)=x^{*}$ ), meaning that we cannot generally infer the stability of individual responses from the stability e.g. of the average response. However, we can imagine such a failure to be a rare event. As an illustration, consider a symmetric one-dimensional linear game with $\varphi^{j}\left(x_{-j}\right)=a \sum_{i \neq j} x_{i}+b$ and equilibrium $x^{*}=\left(x_{1}^{*}, \ldots, x_{1}^{*}\right)$ satisfying $x_{1}^{*}=a(N-1) x_{1}^{*}+b$. Let $Q^{t}=\sum_{j} x_{j}^{t}$ and $z^{t}=Q^{t}-Q^{*}$. As $z^{t}=(a(N-1))^{t} z^{0}=(a(N-1))^{t}\left(\sum_{j} x_{j}^{0}-Q^{*}\right)$, observe that $Q^{t} \rightarrow Q^{*}$ but $x^{t} \nrightarrow x^{*}$ is possible only if $\left(\sum_{j} x_{j}^{0}=Q^{*}\right)$, which does not occur almost surely as $\left\{x \in \mathbb{R}^{n}: \sum_{j=1}^{n} x_{j}=Q^{*}\right\}$ is a zero-measure Lebesgue set.

\section{Conclusion}

This paper has examined the mathematical connections between the time-honored notion of best-response dynamics and uniqueness of equilibria in case of sum-aggregative or symmetric games. It was shown that for sum-aggregative games with equilibrium complements uniqueness and local stability are the same properties: If there is a unique equilibrium, it must be locally contraction-stable and, vice-versa, if every critical point satisfies contraction-stability the game possesses exactly one equilibrium. In case of equilibrium substitutes, local stability of 
equilibrium candidate points implies uniqueness, but not vice-versa. A similar result holds in symmetric games. A symmetric game with equilibrium complements has exactly one symmetric equilibrium if and only if it is contraction-stable. In this case the symmetric equilibrium is also sequentially or gradient stable as well as locally dominance-solvable. Moreover, if best-replies have slopes larger than -1 , the equilibrium is unique (without strategies e.g. being complements everywhere). If strategies are equilibrium substitutes, uniqueness of the symmetric equilibrium does not imply contraction-stability, and contraction-stability is more restrictive than gradient stability. It was illustrated that if players update their response sequentially rather than simultaneously this difference vanishes. This is an interesting result as it suggests that with substitutes, the level of awareness or attention the players devote to a game influences whether or not a self-restoring equilibrium dynamic can be expected to prevail. Furthermore, it was shown that in sum-aggregative symmetric index games with equilibrium substitutes (such as the Cournot or Tullock game) the symmetric equilibrium necessarily is unique and sequentially (or gradient) stable. Finally, the response-dynamics induced by aggregate-taking behavior was compared to the conventional Nash response-dynamics. While in complete generality a comparison with respect to stability produces no clear-cut results due to the presence of two potentially countervailing effects, it was shown that in linear symmetric games contraction-stability of the Nash response dynamics always implies the stability of the aggregate-taking dynamics in case of complements, and vice-versa in case of substitutes.

UNIVERSITY OF ZURICH

HARVARD UNIVERSITY

\section{Proofs}

\subsection{Proof of theorem 1}

$" \Rightarrow "$. Let $q \equiv \sup _{x \in U,|v|_{X}=1}|\partial \phi(x) \cdot v|_{Y}=\sup _{x \in U}\|\partial \phi(x)\|_{|\cdot|_{X}}<1$. Because $U$ is an open, convex set, the mean value theorem gives for any $x, x^{\prime} \in U$ :

$$
\begin{aligned}
\left|\phi(x)-\phi\left(x^{\prime}\right)\right|_{Y} & \leq \sup _{0 \leq t \leq 1,|v|_{X}=1}\left(\left|\partial \phi\left(x+t\left(x^{\prime}-x\right)\right) \cdot v\right|_{Y}\right)\left|x-x^{\prime}\right|_{X} \\
& \leq q\left|x-x^{\prime}\right|_{X}
\end{aligned}
$$


Hence $\phi$ is a contraction on $U$. Let $x \in \partial \bar{U}, x^{\prime} \in \bar{U}$ and take any two sequences $\left(x_{n}\right),\left(x_{n}^{\prime}\right)$ in $U$ with $x_{n} \rightarrow x$ and $x_{n}^{\prime} \rightarrow x^{\prime}$. Because of $\left|\phi\left(x_{n}\right)-\phi\left(x_{n}{ }^{\prime}\right)\right|_{Y} \leq q\left|x_{n}-x_{n}{ }^{\prime}\right|_{X}$ continuity implies $\left|\phi(x)-\phi\left(x^{\prime}\right)\right|_{Y} \leq q\left|x-x^{\prime}\right|_{X}$ which shows that $\phi$ is a contraction on $\bar{U}$.

$" \Leftarrow "$. Suppose $\exists q<1$ such that $\left|\phi(x)-\phi\left(x^{\prime}\right)\right|_{Y} \leq q\left|x-x^{\prime}\right|_{X} \forall x, x^{\prime} \in \bar{U}$. Take an arbitrary $x \in U$ and an arbitrary $v \in \mathbb{R}^{n}$ with $|v|_{X}=1$. Then for any $x^{\prime} \in U$ with $x^{\prime} \neq x$ there exists $t \in \mathbb{R}$ such that $x^{\prime}=x+t v$. Then $q \geq \frac{\left|\phi(x)-\phi\left(x^{\prime}\right)\right|_{Y}}{\left|x-x^{\prime}\right|_{X}}=\frac{|\phi(x+t v)-\phi(x)|_{Y}}{|t|}$. As $\phi$ is Frechet-differentiable on $U$, the directional derivatives exist. Hence

$$
q \geq \lim _{t \rightarrow 0} \frac{|\phi(x+t v)-\phi(x)|_{Y}}{|t|}=\left|\lim _{t \rightarrow 0}\left(\frac{\phi(x+t v)-\phi(x)}{t}\right)\right|_{Y}=|\partial \phi(x) \cdot v|_{Y}
$$

holds for any $x \in U$ and, as $v$ was arbitrary up to $|v|_{X}=1$, we get $\sup _{x \in U,|v|_{X}=1}|\partial \phi(x) \cdot v|_{Y} \leq q<1$.

\subsection{Proof of corollary 1}

$" \Rightarrow$ Let $\sup _{x \in U} \rho(\partial \phi(x))=\delta<1$ and note that continuity of $\rho(\partial \phi(x))$ implies that $\exists \varepsilon>0$ such that $\rho(\partial \phi(x))<\delta+\varepsilon<1$ holds on $\bar{U}$. Then, by fact $3, \forall x_{0} \in \bar{U}$ there exists a matrix norm $\|\cdot\|_{\left(x_{0}\right)}$ such that $\left\|\partial \phi\left(x_{0}\right)\right\|_{\left(x_{0}\right)}<\delta+\varepsilon$. Continuity of $\partial \phi(x)$ asserts the existence of an open neighborhood $B\left(x_{0}\right) \subset \bar{U}$ such that $\|\partial \phi(x)\|_{\left(x_{0}\right)}<\delta+\varepsilon$ for any $x \in B\left(x_{0}\right)$. Because $\bar{U}$ is compact and $\bigcup_{x \in \bar{U}} B(x)$ covers $\bar{U}$ there exists a finite subcover $\bigcup_{j=1}^{n} B\left(x_{j}\right)$, and $\|\partial \phi(x)\|_{\left(x_{j}\right)}<\delta+\varepsilon$ for any $x \in B\left(x_{j}\right)$. Further, $\|\cdot\| \equiv \max \left\{\|\cdot\|_{\left(x_{1}\right)}, \ldots,\|\cdot\|_{\left(x_{n}\right)}\right\}$ is a matrix norm such that $\|\partial \phi(x)\|<\delta+\varepsilon$ holds for any $x \in \bar{U}$ and the claim follows from fact 2 and theorem 1 .

$" \Leftarrow "$ Follows from theorem 1 and fact 3 .

\subsection{Proof of theorem 2}

$" \Rightarrow "$. Define $\sigma(x) \equiv\left\|\partial \phi\left(x^{*}\right)\right\|_{|\cdot|}=\sup _{|v|=1}|\partial \phi(x) \cdot v|$. Hence $q \equiv \sigma\left(x^{*}\right)<1$. As $\sigma$ is continuous at $x^{*}$ there exists a closed $\delta$-ball $\overline{\mathbb{B}}\left(x^{*}, \delta\right) \equiv V, \delta>0$, such that $\sigma(x)<1 \forall x \in V$. Let $Q \equiv \sup _{x \in V} \sigma(x)<1$. Then theorem 1 implies that $\phi$ is a contraction on $V$, i.e. $\left|\phi(x)-\phi\left(x^{\prime}\right)\right| \leq$ $Q\left|x-x^{\prime}\right|$ for $x, x^{\prime} \in V$, and $\left|\phi(x)-\phi\left(x^{*}\right)\right| \leq Q \delta<\delta$ shows that $\phi(V) \subset V$.

$" \Leftarrow "$. Follows from theorem 1. For the last claim note that, using the definition of $\left(x^{t}\right)$, as 
$\phi \in \mathcal{C}(V, V)$ we inductively obtain from $\left|x^{k+1}-x^{k}\right| \leq q\left|x^{k}-x^{k-1}\right|, q=\left\|\partial \phi\left(x^{*}\right)\right\|_{|\cdot|}$, that $\left|x^{k}-x^{t}\right| \leq \frac{(q)^{t}-(q)^{k}}{1-q}\left|x^{1}-x^{0}\right| \leq \frac{(q)^{t}}{1-q}\left|x^{1}-x^{0}\right|$, where $k>t \geq 0$. This shows that $\left(x_{t}\right)$ is Cauchy, hence convergent by the completeness of $V$, and $\lim _{t \rightarrow \infty} x_{t}=\lim _{t \rightarrow \infty} \phi\left(x^{t}\right)=x^{*}$, where the last statement uses continuity of $\phi$ on $V$ and the fact that $\phi$ has exactly one FP on $V$.

\subsection{Proof of corollary 3}

$" \Rightarrow "$. By presupposition there is $|\cdot|$ and $q<1$ such that $\left|L(x)-L\left(x^{\prime}\right)\right| \leq q\left|x-x^{\prime}\right|$ for any $x, x^{\prime} \in \mathbb{R}^{n}$ or equivalently $\left|\partial \phi\left(x^{*}\right) v\right| \leq q|v|, v \in \mathbb{R}^{n}$. Hence also $\left\|\partial \phi\left(x^{*}\right)\right\|_{|\cdot|} \leq q<1$, and the claim follows from theorem 2. "Æ". If $x^{*}$ is contraction-stable, then $\left\|\partial \phi\left(x^{*}\right)\right\|_{|\cdot|}<1$ for some $|\cdot|$ because of theorem 2. The claim then follows from $\left|L(x)-L\left(x^{\prime}\right)\right|=\left|\partial \phi\left(x^{*}\right)\left(x-x^{\prime}\right)\right| \leq$ $\left\|\partial \phi\left(x^{*}\right)\right\|\left|x-x^{\prime}\right|$. To see the last claim, let $x_{t+1}=\phi \circ \phi \circ \ldots \phi\left(x_{0}\right) \equiv z\left(x_{0}\right)$. Linearizing $z$ at $x^{*}$ gives $z\left(x_{0}\right) \cong x^{*}+\partial \phi\left(x^{*}\right)^{t}\left(x_{0}-x^{*}\right)$. The suggested bound then follows as $\left|x_{t+1}-x^{*}\right| \cong$ $\left|\partial \phi\left(x^{*}\right)^{t}\left(x_{0}-x^{*}\right)\right| \leq \rho\left(\partial \phi\left(x^{*}\right)\right)^{t}\left|x_{0}-x^{*}\right|$ (use submultiplicativity and facts 2 and 3 ).

\subsection{Proof of corollary 5}

For a vector $v$ let $v^{+} \equiv\left(\left|v_{i}\right|\right)$ denote the vector of the absolute values of the components of $v$. Similarly, if $M$ is a matrix then $M^{+}$denotes the matrix of absolute values of the components of $M$. The triangle inequality implies $(M v)^{+} \leq M^{+} v^{+}$. Let $H^{g}=\frac{\partial^{2} \Pi^{g}(x)}{\partial x_{g} \partial x_{g}^{T}}$ denote the Hessian of $\Pi^{g}(x)$. As $H\left(x^{*}\right)$ has a dominant negative diagonal, the matrix $H^{g}$ is negative definite and thus invertible. Hence the IFT gives for $j \neq g$ and $1 \leq i \leq 2$ :

$$
\left(\frac{\partial \varphi^{g}}{\partial x_{j i}}\right)^{+}=\left(-\left(H^{g}\right)^{-1}\left(\frac{\partial \nabla_{g} \Pi^{g}}{\partial x_{j i}}\right)\right)^{+} \leq\left(\left(H^{g}\right)^{-1}\right)^{+}\left(\frac{\partial \nabla_{g} \Pi^{g}}{\partial x_{j i}}\right)^{+}
$$

which further implies

$$
\sum_{j \neq g} \sum_{i=1}^{k}\left(\frac{\partial \varphi^{g}}{\partial x_{j i}}\right)^{+} \leq\left(\left(H^{g}\right)^{-1}\right)^{+} z_{g}
$$

where $z_{g}=\sum_{j \neq g} \sum_{i=1}^{2}\left(\frac{\partial \nabla_{g} \Pi^{g}}{\partial x_{j i}}\right)^{+}$. Let $\hat{H}^{g}$ be the matrix derived from $H^{g}$ by taking the absolute values of each off-diagonal element. Because $H\left(x^{*}\right)$ has a dominant negative diagonal it follows 
that $-\hat{H}^{g} \cdot 1>z_{g}$. Further simple algebra shows that

$$
\left(\left(H^{g}\right)^{-1}\right)^{+}=\left(-\hat{H}^{g}\right)^{-1} \geq 0
$$

Hence we get

$$
\left(-\hat{H}^{g}\right)^{-1}\left(-\hat{H}^{g}\right) \cdot 1>\left(-\hat{H}^{g}\right)^{-1} z_{g} \geq\left(\left(H^{g}\right)^{-1}\right)^{+} z_{g}
$$

which by (11) gives $I \cdot 1>\sum_{j \neq g} \sum_{i=1}^{2}\left(\frac{\partial \varphi^{g}}{\partial x_{j i}}\right)^{+}$for any $g=1, \ldots, N$. Thus we may conclude that $R_{m}<1$ holds $\forall m$.

Remark: The above proof can be generalized to the case $k>2$, the main problem being expression (12). Using laborious Laplace expansions (and the triangle inequality) it follows that

$$
\left(\left(H^{g}\right)^{-1}\right)^{+} \leq\left(\left(-\hat{H}^{g}\right)^{-1}\right)^{+}=\left(-\hat{H}^{g}\right)^{-1}
$$

The equality between the second and third term follows from the fact that $-\hat{H}^{g}$ is an invertible matrix with non-positive off-diagonal elements (a $Z$-matrix) and it is known that $\left(-\hat{H}^{g}\right)^{-1}$ must be non-negative (see e.g. Mckenzie (1959)). The rest of the proof remains the same.

\subsection{Proof of lemma 2}

The case where $\rho(A)=0$ is trivial so suppose $\rho(A)>0$. Note that $\alpha \in \sigma(M)$ if and only if

$$
M V=\left(\begin{array}{c}
A\left(v_{2}+\ldots+v_{N}\right) \\
A\left(v_{1}+v_{3}+\ldots+v_{N}\right) \\
\vdots \\
A\left(v_{1}+\ldots+v_{N-1}\right)
\end{array}\right)=\alpha V \quad V \neq 0
$$

where $V=\left(v_{j}\right)_{j=1}^{N}$ and each $v_{j}$ is itself a $k$-vector. If $A v=\lambda v$ then setting $v_{j}=\frac{v}{N-1}$ shows that $\lambda(N-1) \in \sigma(M)$, as every row of (13) reads $A v=\frac{\alpha}{N-1} v$. This is true for every $\lambda \in \sigma(A)$ (with the corresponding eigenvector), hence we have found $k \mathrm{EV}$ of $M$. To find the other $\mathrm{EV}$ of $M$, first set $v_{1}=v, v_{2}=-v$ and $v_{3}=\ldots=v_{N}=0$. Then, the first row of (13) reads $A(-v)=\alpha v$, the second row is $A v=\alpha(-v)$ and all other rows are $0=0$. Hence we found $k$ further EV 
of $M$, and they take on the values $-\sigma(A)$. Continuing by setting $v_{1}=v$ and $v_{3}=-v$ leaving all other coordinates zero gives the next $k \mathrm{EV}$ (note that the so constructed eigenvectors of $M$ are linearly independent). Proceeding in this manner shows that all EV of $M$ must be either $(N-1) \lambda$ or $-\lambda, \lambda \in \sigma(A)$.

\subsection{Proof of theorem 4}

Lemma 1 implies that $\operatorname{Det}(-H)=\prod_{j}\left(-\Pi_{11}^{j}\right)\left(1+b_{j}\right)\left(1-\sum_{i} \frac{b_{i}}{1+b_{i}}\right)$ if $b_{j} \neq-1$ and $\operatorname{Det}(-H)=$ $\prod_{j}\left(-\Pi_{11}^{j}\right) \prod_{i \neq j}\left(1+b_{j}\right)$ if $b_{j}=-1$. Hence strong quasiconcavity and $b_{j}(x) \geq 0 \mathrm{imply} \operatorname{Det}(-H(x))>$ 0 on $C r$ if and only if $\sum \frac{b_{j}(x)}{1+b_{j}(x)}<1$, and the first claim follows from theorem 3 and corollary 6. To prove the second claim, suppose that $b_{j} \leq 0 \forall j$ at any critical point, and note that if $b_{j} \in(-1,0) \forall j$ at critical points, then the equilibrium is necessarily unique independent of whether or not $x \in C r$ is contraction-stable. Further, if $x \in C r$ is contraction-stable, we can have $b_{j}(x) \geq-1$ for at most one $j$, so henceforth suppose that $b_{1}(x) \leq-1, b_{j}(x) \in(-1,0]$ for $j>1$ and $x \in C r$ is contraction-stable. If $b_{1}(x)=-1$, then $\operatorname{Det}(-H(x))>0$ as $\left(1+b_{j}(x)\right)>0$ for $j>1$. It remains to show that $b_{1}(x)<-1$ also implies $\operatorname{Det}(-H(x))>0$. But $\operatorname{Det}(-H(x))>0$ holds under the previous assumptions if and only if $\sum_{j} \frac{b_{j}(x)}{1+b_{j}(x)}>1$. Let $z_{j}(x) \equiv \frac{b_{j}(x)}{b_{j}(x)-1}$ and note that $x$ is contraction-stable if and only if $\sum_{j} z_{j}(x)<1$. Now set $z_{1}=\varepsilon-\sum_{j>1} z_{j}$, where $\varepsilon<1$ by contraction-stability. Note that $z_{1} \in\left(\frac{1}{2}, 1\right), z_{j} \in\left[0, \frac{1}{2}\right)$ for any $j>1$ and $\sum_{j>1} z_{j}<\frac{1}{2}$. We have $\operatorname{Det}(-H)>0$ if

$$
\frac{b_{1}}{1+b_{1}}+\sum_{j>1} \frac{b_{j}}{b_{j}+1}=\frac{\sum_{j>1} z_{j}-\varepsilon}{2\left(\sum_{j>1} z_{j}-\varepsilon\right)+1}+\sum_{j>1} \frac{z_{j}}{2 z_{j}-1} \equiv \zeta(\varepsilon)>1
$$

As $z_{1}>\frac{1}{2}$ the first summand is larger than 1 and strictly decreasing in $\varepsilon$. Hence it suffices to show that $\zeta(1) \geq 1$, which trivially holds if $z_{j}=0$ for all $j>1$. Assuming $z_{N} \in\left(0, \frac{1}{2}\right)$ and using derivatives it is straightforward to verify that $(0, \ldots, 0)=\underset{z_{2}, \ldots, z_{N-1}}{\operatorname{argmin}} \zeta(1)$ for $z_{2}, \ldots, z_{N-1} \in[0,1 / 2)$ and $\sum_{j=2}^{N-1} z_{j} \leq \frac{1}{2}-z_{N}$, which implies $\zeta(1) \geq \frac{z_{N}-1}{2\left(z_{N}-1\right)+1}+\frac{z_{N}}{2 z_{N}-1}=1$ for any $z_{N} \in\left(0, \frac{1}{2}\right)$, which completes the proof. 


\section{References}

Alos-Ferrer, C. and Ania, A. B. (2005). The evolutionary stability of perfectly competitive behavior. Economic Theory, 26:497-516. 10.1007/s00199-004-0474-8.

Cournot, A. (1897). Researches into the Mathematical Principles of the Theory of Wealth. New York: McMillan.

Dastidar, K. G. (2000). Is a unique cournot equilibrium locally stable? Games and Economic Behavior, 32:206-218.

Diewert, W. E., Avriel, M., and Zang, I. (1981). Nine kinds of quasiconcavity and concavity. Journal of Economic Theory, 25:397 - 420.

Dixit, A. (1986). Comparative statics for oligopoly. International Economic Review, 27:107-122.

Gabay, D. and Moulin, H. (1980). On the uniqueness and stability of nash-equilibria in noncooperative games. In Bensoussan, A., Kleindorfer, P., and Tapiero, C. S., editors, Applied Stochastic Control in Econometrics and Management Science. North-Holland.

Grossman, G. M. and Shapiro, C. (1984). Informative advertising with differentiated products. The Review of Economic Studies, 51:63-81.

Grossmann, M., Hefti, A. M., and Lang, M. (2012). Aggregative contests and ex-post heterogeneity. ISU working paper 161.

Hadar, J. (1966). Stability of oligopoly with product differentiation. The Review of Economic Studies, 33:57-60.

Hefti, A. M. (2011). On uniqueness and stability of symmetric equilibria in differentiable symmetric games. Econ Working Paper 18.

Horn, R. A. and Johnson, C. R. (1985). Matrix Analysis. Cambridge University Press.

Konrad, K. (2009). Strategy and Dynamics in Contests. Oxford University Press.

Mckenzie, L. (1959). Matrices with dominant diagonals and economic theory. In Arrow, K., editor, Mathematical Methods in the Social Sciences. Stanford University Press. 
McManus, M. and Quandt, R. E. (1961). Comments on the stability of the cournot oligipoly model. The Review of Economic Studies, 28:136-139.

Moulin, H. (1984). Dominance solvability and cournot stability. Mathematical Social Science, $7: 83-102$.

Nitecki, Z. (1971). Differentiable Dynamics. Cambridge: MIT Press.

Okuguchi, K. and Yamazaki, T. (2008). Global stability of unique nash equilibrium in cournot oligopoly and rent-seeking game. Journal of Economic Dynamics and Control, 32:1204-1211.

Salop, S. (1979). Monopolistic competition with outside goods. The Bell Journal of Economics, 10:141-156.

Vives, X. (1999). Oligopoly pricing. Cambridge: MIT Press. 